\title{
Opioid Withdrawal and Restless Legs Syndrome
}

\author{
Eun Jin Park and Young-Min Park \\ Department of Psychiatry, Ilsan Paik Hospital, Inje University College of Medicine, Goyang, Korea
}

\begin{abstract}
Opioids are widely used for treating pain and severe treatment-resistant restless leg syndrome (RLS). However, reports of opioid withdrawalinduced RLS have recently increased. Various opioids, including fentanyl, oxycodone, tramadol, dextropropoxyphene, and heroin, induce withdrawal RLS, which is an agonizing illness. There are various hypotheses around opioid withdrawal-induced RLS. First, opioids are associated with dopaminergic action in the mesolimbic pathway. The persistent occupancy of opioids in $\mu$-receptors increases dopamine release, but eventually leads to reduced sensitivity or down-regulation of dopamine receptors. The abrupt depletion of dopamine occurs after opioid use cessation, producing RLS. Another explanation is that long-term stimulation of opioid receptors induces the activation of adenylyl cyclase and protein kinase $A$, which activates tyrosine hydroxylase involved with dopamine synthesis and the reduction of endogenous opiates. Discontinuing opioid use can also lead to the decrement of dopamine synthesis, producing RLS. Additionally, opioid deficiency, regardless of dopamine, is a pathogenesis pathway in opioid withdrawal-induced RLS. No treatment for opioid withdrawal-induced RLS has been established. However, general RLS treatments also apply to opioid withdrawal-induced RLS, including the use of ropinirole, clonazepam, pregabalin, and opioids. Clinicians must be aware of RLS development shortly after the cessation of opioid treatment for pain control/opioid addiction.

Key Words: Opioids; Restless leg syndrome; Withdrawal symptoms; Fentanyl; Oxycodone; Tramadol

Received: November 21, 2020 Accepted: November 26, 2020

Corresponding author: Young-Min Park, MD, PhD, Department of Psychiatry, Ilsan Paik Hospital, Inje University College of Medicine, 170 Juhwa-ro, Ilsanseo-gu, Goyang 10380, Korea.

Tel: 82-31-910-7260, Fax: 82-31-910-7268, E-mail: medipark@hanmail.net

(a) This is an Open Access article distributed under the terms of the Creative Commons Attribution Non-Commercial License (https://creativecommons.org/licenses/bync/4.0) which permits unrestricted non-commercial use, distribution, and reproduction in any medium, provided the original work is properly cited.
\end{abstract}

\section{INTRODUCTION}

Opioids are widely used for pain treatment but need to be handled with care because of their dependency risk and severe withdrawal symptoms. Opioids are also used as a treatment for severe, treatment-resistant restless legs syndrome (RLS) [1]. In recent years, RLS development following the discontinuation of opioid use has been reported. In this review, we describe various RLS cases that occurred after the cessation of opioid use. The following cases had no previous RLS history, no primary RLS, and no other causes of secondary RLS except for their opioid withdrawal.

\section{CASES}

\section{Fentanyl}

Fentanyl is an opioid analgesic. Specifically, transdermal fentanyl is a well-used and highly effective form of opioid analgesia for individuals suffering from severe chronic pain [2]. It is also an opioid that is widely abused and results in significant withdrawal symptoms. Fentanyl withdrawal can lead to restlessness, nausea, vomiting, sweating, diarrhea, and myoclonus, all in addition to RLS [3-5].

We previously reported on a 58-year-old male with fentanyl patch withdrawal-induced RLS [5]. He utilized the patch for two months after having surgery on his right shoulder. After the discontinuation of the patch, he complained of severe insomnia. He experienced an unpleasant sensation in both legs that was aggravated in the nighttime, and which could only be improved only by moving his legs. The patient thus met all the diagnostic criteria for RLS [6]. He readministered the patch because of his insomnia, after which his RLS symptoms disappeared. His RLS symptoms recurred repeatedly whenever he stopped using the patch. His orthopedic surgeon then referred him for psychiatric consultation. The patient had no disease related to secondary RLS. He was also taking no medication except for the patch. Finally, his RLS symptoms disappeared following the commencement of ropinirole/ clonazepam therapy. 


\section{Tramadol}

Tramadol is an analgesic which has $\mu$-opioid receptor agonist effects and norepinephrine and serotonin-reuptake inhibition as a synthetic codeine analogue [7].

We also reported on a 38-year-old male with tramadol withdrawal-induced RLS [8]. He had a large amount of pleural effusion, with pain. Chest-tube placement with both tramadol (150 $\mathrm{mg} /$ day) and antibiotics lasted for several weeks. His pain was largely improved after tramadol treatment. However, after undergoing tramadol discontinuation, he complained of an unpleasant crawling sensation in the feet that aggravated during both rest and at night, and which could only be lessened with movement. He suffered from severe insomnia and would wander around his bed every night. Tramadol was readministered to confirm that this was a case of tramadol withdrawal-induced RLS, which dramatically resolved his RLS symptoms. Whenever he stopped tramadol, his RLS symptoms repeated again, leading him to take it; eventually, tramadol was changed into clonazepam and ropinirole, which alleviated his RLS symptoms. Clonazepam and ropinirole were tapered off gradually after several weeks, with there then being no recurrences of any RLS symptoms.

A group of clinicians have reported another case of tramadol withdrawal-induced RLS [9]. Their patient was a 45-year-old female with no previous history of musculoskeletal pain when she was admitted to the pain clinic. She expressed significant relief following the intake of liquid tramadol, at a dose of $50-100 \mathrm{mg}$, which she usually took daily, in the morning, for 12 months. While on vacation in a foreign country, she ran out of tramadol and, after nine days, she then developed RLS. Benzodiazepine and dextromethorphan treatment effectively alleviated her RLS symptoms.

In addition to tramadol withdrawal, there were two case reports in which RLS symptoms developed during the actual treatment of RLS using tramadol $[10,11]$. In the first case, four patients, who had received tramadol for two to four years for RLS treatment, were taken off the medication due to the reappearance of their RLS symptoms (a phenomenon called augmentation). The tramadol dosage ranged from $100 \mathrm{mg} /$ day to $300 \mathrm{mg} /$ day. In addition, all four patients experienced an acute aggravation of their RLS symptoms due to tramadol withdrawal. However, there was also subsequent improvement in their symptoms when they returned to the pre-treatment state after about four to seven days of being off tramadol. In the second case, one female patient received an additional $50 \mathrm{mg}$ of tramadol because of an insufficient treatment response to clonazepam. One year later, she experienced an aggravation of her RLS symptoms, with its new onset occurring during the day. Two months after her discontinuation of tramadol, following the administration of niaprazine, her RLS symptoms had mostly improved.

\section{Oxycodone}

Oxycodone is an alternative treatment option for RLS as a semisynthetic opioid. The $\kappa$-opioid and the $\mu$-opioid receptor mediate its analgesic effect, but this has not been clearly confirmed. Oxy- codone withdrawal-induced RLS has also been reported as follows [12].

A 42-year-old male patient was medicated with an analgesic (oxycodone $60 \mathrm{mg}$ bid) for 10 weeks with embolization because of a hepatocellular carcinoma rupture and hematoperitoneum with pain. Thereafter, his pain was relieved, and he arbitrarily stopped taking the oxycodone. Seven days later, he complained of a tickling sensation in both his legs. This sensation began in the evening and become increasingly aggravated until midnight. These symptoms worsened at rest and were mitigated only when he moved his legs. His symptoms satisfied with all the diagnostic criteria for RLS. The severe abdominal pain recurred, and he restarted taking the oxycodone at his own discretion. The irresistible and tickling sensations in both his legs then dramatically disappeared.

\section{Heroin}

Heroin is an addictive drug made from morphine [13]. It has been used as a recreational drug and is often abused. Heroin withdrawal-induced RLS has also been reported as follows.

A 34-year-old male patient with a five year history of heroin addiction [14]. Two days after he discontinued his use of heroin, he complained of all four of RLS's diagnostic symptoms. L-dopa and subsequent naltrexone treatment were then utilized and relieved both his RLS symptoms and heroin addiction. In another study, 19 patients with opioid dependence, who were admitted to a hospital for detoxification, were enrolled [15]. After abstaining from opioids, 10 of the 19 patients developed RLS symptoms. Six of these 10 patients had taken heroin, with the rest of them having taken dextropropoxyphene $(n=2)$, tramadol $(n=1)$, pentazocine $(n=1)$, and crude opium ( $n=1)$. Their RLS symptoms appeared after a mean of 1.7 days following them ceasing their use of opioids. Pregabalin lead to significant improvement in their RLS symptoms and overall sleep quality.

\section{Dextropropoxyphene}

Dextropropoxyphene is a weak synthetic opioid [16]. One group of investigators reported on three dextropropoxyphene withdrawal-induced RLS cases [17]. Three patients were studied, who were all dextropropoxyphene abusers for several years. They were hospitalized and had received the standard detoxication program. After undergoing an abstinence period from dextropropoxyphene, they developed RLS symptoms. However, ropinirole treatment then lead to improvements of and relief from their RLS symptoms.

\section{PATHOPHYSIOLOGY OF OPIOID WITHDRAWAL-INDUCED RESTLESS LEGS SYNDROME}

Opioid medications have been reported to alleviate RLS symptoms [1]. Opioid regulates dopaminergic action in the mesolimbic pathway [18]. Additionally, opioid withdrawal can cause RLS [15]. Thus, opioid is associated with RLS, although the exact rela- 
tionship is not clear. All cases that we reviewed had the temporal pathway between the discontinuation of opioids and the subsequent development of RLS. All these cases had no idiopathic RLS, nor did they possess any other cause of secondary RLS, including an iron deficiency, renal problems, thyroid diseases, etc. In addition, the re-administration of the discontinued opioids led to the improvement of opioid withdrawal-induced RLS. However, in some cases, the RLS symptoms persisted, although other opioid withdrawal symptoms disappeared. Thus, opioid withdrawal is clearly a precipitating factor in RLS.

There is one hypothesis for the relationship between opioid withdrawal and the development of RLS. Opioids usually have a high affinity with $\mu$-opioid receptors. Agonism of $\mu$-opioid receptors leads to increased dopamine release in several brain regions [19]. The persistent occupancy of opioids in these $\mu$-receptors may initially increase the release of dopamine, but eventually leads to a reduced sensitivity or down-regulation of dopamine receptors [20]. Thus, the abrupt depletion of dopamine release may occur after opioid discontinuation, producing RLS. Another explanation is that the long-term stimulation of opioid receptors induces the activation of adenylyl cyclase and protein kinase $\mathrm{A}$, which subsequently cause the activation of tyrosine hydroxylase, which is involved with dopamine synthesis and the reduction of endogenous opiates [6]. Thus, opioid discontinuation leads to decreased activity of adenylyl cyclase and protein kinase A, causing a state of dopamine and opioid depletion in the brain [1].

In addition, deficiency of opioids, regardless of dopamine, is likely one dimension of the pathogenesis of opioid withdrawalinduced RLS $[21,22]$. In a post-mortem study, beta endorphin was found to be decreased in the thalamus of RLS patients with intact dopaminergic activity [22]. These results suggest a state of opioid depletion in opioid withdrawal-induced RLS, irrespective of the dopamine system.

\section{TREATMENT OF OPIOID WITHDRAWAL RESTLESS LEGS SYNDROME}

An effective treatment for opioid withdrawal-induced RLS remains to be established. However, there are general treatment principles that improve the symptoms of RLS. Most cases show improvement after the use of ropinirole and/or benzodiazepines, such as clonazepam. However, a recent study found that nine patients with opioid withdrawal-induced RLS had good responses to pregabalin treatment [15]. The mean dosage of pregabalin was $150 \mathrm{mg} /$ day, while one participant received a mean dosage of 75 mg/day.

In one case, pramipexole and pregabalin treatment did not alleviate RLS symptoms [12]. The symptoms did, however, disappear shortly after the re-administration of oxycodone/naloxone that was previously used for pain control. Thus, opioid therapy should be also considered as an option for the treatment of opioid withdrawal-induced RLS.

\section{CONCLUSION}

Opioids are widely used as both pain and RLS treatment, but should be handled with care because of their dependency risk and severe withdrawal symptoms. Opioids are associated with RLS, although this exact relationship is not clear. In recent years, RLS development shortly after the discontinuation of opioids has been reported in various case studies. Opioid withdrawal-induced RLS, although relatively uncommon, is an agonizing illness that severely impacts peoples' quality of sleep. Thus, clinicians must be aware of the occurrence of RLS shortly after the cessation of opioid treatment for pain control/opioid addiction.

\section{Acknowledgments}

This study was supported by a grant from the National Research Foundation of Korea (NRF), funded by the Ministry of Science and ICT (2020R1F1A1073188).

\section{Conflicts of Interest}

The authors have no potential conflicts of interest to disclose.

\section{Author Contributions}

Conceptualization: Young-Min Park. Data curation: Young-Min Park. Project administration: Young-Min Park. Resources: Eun Jin Park, Young-Min Park. Supervision: Young-Min Park. Writing-original draft: Eun Jin Park, Young-Min Park. Writing-review \& editing: Eun Jin Park, Young-Min Park.

\section{ORCID iDs}

Eun Jin Park (1D

https://orcid.org/0000-0003-4046-1517

Young-Min Park (1)

https://orcid.org/0000-0002-4993-1426

\section{REFERENCES}

1. Gonzalez-Latapi P, Malkani R. Update on restless legs syndrome: from mechanisms to treatment. Curr Neurol Neurosci Rep 2019;19:54.

2. Langford NJ. Fentanyl patches: use and misuse. Acute Med 2020;19:90-96.

3. Zenz M, Donner B, Strumpf M. Withdrawal symptoms during therapy with transdermal fentanyl (fentanyl TTS)? J Pain Symptom Manage 1994;9:5455.

4. Higgs CM, Vella-Brincat J. Withdrawal with transdermal fentanyl. J Pain Symptom Manage 1995;10:4-5.

5. Park YM, Cho JH, Lim YS, Lee HJ, Kang SG, Kim L. The withdrawal from TDF therapy could induce transient RLS. Prog Neuropsychopharmacol Biol Psychiatry 2010;34:419-420.

6. Allen RP, Picchietti D, Hening WA, Trenkwalder C, Walters AS, Montplaisi J. Restless legs syndrome: diagnostic criteria, special considerations, and epidemiology. A report from the restless legs syndrome diagnosis and epidemiology workshop at the National Institutes of Health. Sleep Med 2003;4:101119.

7. Grond S, Sablotzki A. Clinical pharmacology of tramadol. Clin Pharmacokinet 2004;43:879-923.

8. Park YM, Park HK, Kim L, Lee HJ, Kang SG. Acute-withdrawal restless legs syndrome following abrupt cessation of short-term tramadol. Psychiatry Investig 2014;11:204-206.

9. Freye E, Levy J. Acute abstinence syndrome following abrupt cessation of long-term use of tramadol (Ultram): a case study. Eur J Pain 2000;4:307-311. 
10. Earley CJ, Allen RP. Restless legs syndrome augmentation associated with tramadol. Sleep Med 2006;7:592-593.

11. Vetrugno R, La Morgia C, D’Angelo R, Loi D, Provini F, Plazzi G, et al. Augmentation of restless legs syndrome with long-term tramadol treatment. Mov Disord 2007;22:424-427.

12. Baek JH, Han SH, Lee SA. Restless leg syndrome induced by an acute withdrawal of oxycodone. Sleep Med Res 2015;6:84-86.

13. Darke S, Zador D. Fatal heroin 'overdose': a review. Addiction 1996;91:17651772.

14. Scherbaum N, Stüper B, Bonnet U, Gastpar M. Transient restless legs-like syndrome as a complication of opiate withrawal. Pharmacopsychiatry 2003; 36:70-72.

15. Gupta R, Ali R, Ray R. Willis-Ekbom disease/restless legs syndrome in patients with opioid withdrawal. Sleep Med 2018;45:39-43.

16. Barkin RL, Barkin SJ, Barkin DS. Propoxyphene (dextropropoxyphene): a critical review of a weak opioid analgesic that should remain in antiquity. Am J Ther 2006;13:534-542.
17. Ghosh A, Basu D. Restless legs syndrome in opioid dependent patients. Indian J Psychol Med 2014;36:85-87.

18. Leone P, Pocock D, Wise RA. Morphine-dopamine interaction: ventral tegmental morphine increases nucleus accumbens dopamine release. Pharmacol Biochem Behav 1991;39:469-472.

19. Fields HL, Margolis EB. Understanding opioid reward. Trends Neurosci 2015;38:217-225.

20. Walker JM, Thompson LA, Frascella J, Friederich MW. Opposite effects of $\mathrm{mu}$ and kappa opiates on the firing-rate of dopamine cells in the substantia nigra of the rat. Eur J Pharmacol 1987;134:53-59.

21. Hening WA, Walters A, Kavey N, Gidro-Frank S, Côté L, Fahn S. Dyskinesias while awake and periodic movements in sleep in restless legs syndrome: treatment with opioids. Neurology 1986;36:1363-1366.

22. Walters AS, Ondo WG, Zhu W, Le W. Does the endogenous opiate system play a role in the Restless Legs Syndrome? A pilot post-mortem study. J Neurol Sci 2009;279:62-65. 